\title{
Formación para el mundo del trabajo desde la resolución de problemas
}

\section{Training for solve-problems about world of work}

\author{
Antonio Fabregat Pitarch*, Isabel María Gallardo Fernández** \\ *Escuelas de Artesanos-Valencia, **Universitat de Valencia
}

\begin{abstract}
Resumen
Nuestra aportación se concreta en una experiencia de aula realizada en Formación Profesional que se inserta en un planteamiento de intervención educativa que mejore el proceso de enseñanza-aprendizaje desde la resolución de problemas y la implementación de una metodología docente participativa y colaborativa. En nuestra práctica docente optamos por potenciar una formación integral que capacite al alumnado profesionalmente y contribuya, al mismo tiempo, en dotarle de unas competencias personales de saber trabajar en equipo en distintas y variadas situaciones con personas y/ o grupos diversos en la Empresa. El alumnado ha adquirido habilidades/ conocimientos para gestionar compras y resolver problemas.

Palabras clave: [Innovación educativa, Formación Profesional, Resolución de problemas, Trabajo colaborativo.]
\end{abstract}

\section{Resumo}

Nosa contribución asume a forma dunha experiencia de aula feita na formación profesional que insírese nunha visión para a intervención educativa para mellorar o proceso de ensino-aprendizaxe a partir da resolución de problemas ea implementación dunha metodoloxía de ensino participativo e colaborativo. $\mathrm{Na}$ nosa práctica docente, optamos por promover unha formación ampla que permite aos estudantes profesional e contribuír á vez, proporcionar-lle un habilidades persoais para traballar en equipo en diversas situacións da vida coas persoas e / ou en varios grupos na empresa. Os alumnos adquiriron habilidades / coñecemento para xestionar a compra e resolver problemas.

Palabras clave: [Innovación educativa, A formación profesional, Resolución de problemas, Traballo colaborativo.]

\footnotetext{
Abstract

Our contribution takes the form of a classroom experience made in vocational training that is inserted into an approach to educational intervention to improve the teaching-learning process from the resolution of problems and the implementation of a participatory and collaborative teaching methodology. In our teaching practice we opted to promote a comprehensive training that enables students to professionally and contribute at the same time, provide it with a personal skills to work in teams in diverse life situations with people and / or various groups in the company. The students have acquired skills / knowledge to manage purchases and solve problems.

Keywords: [Educational innovation, Vocational training, problem solving, Collaborative work]
}

\section{Introducción}

Esta experiencia surge a través de la observación y análisis de la práctica docente de cursos anteriores en la que el alumnado del Ciclo Formativo de "Gestión Comercial y Marketing" para trabajar los contenidos curriculares de la unidad de competencia 3 aprende a resolver problemas pero sin comprender bien lo que hace. Podríamos decir que aprenden a resolver problemas de forma mecánica desde la memorización de fórmulas y problemas tipo resueltos en el aula. En este caso, se trataba de aprendizajes por repetición en la resolución de problemas sin centrarse en el significado ni en la reflexión del proceso seguido. Básicamente, el alumnado se fijaba en el resultado final. Estábamos ante alumnos/as desmotivados y acostumbrados a estudiar de forma mecánica y repetitiva.

Del análisis de la práctica docente se deduce que los alumnos/as no están motivados por la tarea, simplemente les importaba solucionar bien el problema sin reflexionar sobre el significado de lo que representaba. Así, se quejaban año tras año de la cantidad de fórmulas y datos que habían de aprender sobre la gestión de stocks. Ante esta realidad, los docentes decidimos replantear la metodología de aula desde un enfoque sociocultural de la enseñanza que propicie la consecución de habilidades y estrategias que fomenten el aprender haciendo (Morin, 2011). La Unidad de Competencia 3 se concreta en: gestionar las existencias en almacén, de acuerdo con criterios establecidos, asegurando el nivel de servicio al cliente y la optimización del coste de los stocks.

Somos conscientes que caminamos hacia un paradigma basado en un modelo educativo que supone una nueva definición de las actividades de enseñanzaaprendizaje; fomento del trabajo colaborativo y cooperativo aprovechando las Tecnologías de la sociedad de la Información y del Conocimiento (TIC) y sus posibilidades para desarrollar nuevos modos de aprender; ayudar a los alumnos/as a desarrollar sus capacidades., etc. Tal y como sostienen las especialistas Anijovich y Mora (2010: 23) definimos las estrategias de enseñanza como el conjunto de decisiones que toma el docente para orientar la enseñanza con el fin de promover el aprendizaje de sus alumnos. Se trata de orientaciones generales acerca de cómo enseñar un contenido disciplinar considerando qué queremos que nuestros alumnos comprendan, por qué y para qué. 


\section{Marco teórico de referencia}

Optamos por plantear en el grupo-clase tareas que facilitaran un aprendizaje significativo y relevante tomando como punto de partida los saberes del alumnado (Pérez, 2012; Wells, 2001). Iniciamos esta experiencia desde el dialogo que se genera en el aula para centrar la enseñanza en el alumnado desde la realización de tareas complejas como el diseño y elaboración de mapas conceptuales y el aprendizaje basado en problemas. Estrategias didácticas que permiten al alumnado contextualizar sus aprendizajes y organizar la información dando sentido y coherencia a los temas que estudia e investiga en el Ciclo Formativo (Real Decreto 1651/94 y Ley Orgánica 8/2013) .

Trabajar desde sus conocimientos previos motiva intrínsecamente $\mathrm{y}$ hace participar activamente al alumno/a, ya que comprende lo que hace, por qué lo hace y para qué lo hace. Así, el propio alumno/a desarrolla un conjunto de habilidades cognoscitivas que permiten pensar y reflexionar sobre lo que se hace $\mathrm{y}$ porqué se hace. La idea básica se fundamenta en que el aprendizaje es un proceso activo sobre el cual el discente construye su conocimiento sobre lo que ya sabía y que poco a poco va modificando su estructura mental (Mercer, 1997).

Estas actividades programadas hacen participar activamente al sujeto, ya que son para ellos atractivas y motivadoras. Podemos decir que, el sujeto aprende a través del diseño y puesta en acción de una instrucción estructurada en la que se plantean conflictos cognitivos que con los conocimientos a priori que tenían más los conocimientos adquiridos hacen que el alumno/a encuentre la solución a cada uno de los problemas planteados y asimismo, comprendan su significado (Anijovich y Mora, 2010).

Otro referente teórico en esta experiencia de aula es el Aprendizaje Basado en Problemas (ABP). Sabido es que la metodología ABP centra su aprendizaje en la investigación y reflexión para encontrar la solución ante un problema planteado. Los propios alumnos/as se convierten en protagonistas de su aprendizaje y están más motivados al tratar de resolver un problema planteado vinculado con la realidad tanto social como profesional. El profesor/a ante esta metodología no es el transmisor de información, ni organiza el contenido en una exposición sino que tiene un rol diferente asesorando, guiando, facilitando y tutorizando a sus alumnos/as. Así, facilitamos el que el propio alumnado tome la responsabilidad de aprender (Díaz, 2005).

El cambio metodológico que hemos iniciado en nuestras aulas de Formación profesional tiene un referente en el aprendizaje colaborativo que se convierte en una de las claves de estas prácticas, ya que la ayuda entre iguales hace que cada alumno/a pueda avanzar y superar las dificultades que pueda tener. Los "contenidos" pasan a segundo plano, mientras que "el proceso" se convierte en protagonista como estrategia para conseguir el objetivo principal que es el desarrollo de la competencia profesional y al mismo tiempo desarrollar capacidades de comunicación y cooperación (Cabrera, 2008).
En esta línea de investigación, la implementación del currículo en Formación Profesional supone concebir el aula como un espacio privilegiado para potenciar la autonomía personal, en el que se confíe en las capacidades del alumnado y donde las tareas estén cargadas de intencionalidad educativa. No podemos obviar el valor del diálogo en el aula, que permite la construcción de conocimiento conjunto y la metacognición para recrear las situaciones de aprendizaje. Todo ello constituye la esencia de una enseñanza-aprendizaje relevante y con sentido (Edwards y Mercer, 1987; Woods, 2011).

\section{Contexto y finalidades de la propuesta de trabajo}

Esta experiencia se inserta en un planteamiento de intervención educativa que mejore el proceso de enseñanza-aprendizaje a través de una metodología docente participativa y colaborativa. Se ha llevado a cabo en el contexto de las Escuelas de Artesanos de Valencia durante el curso académico 2014-2015; en concreto, en el Ciclo Formativo de Grado Superior "Gestión Comercial y Marketing” y en el módulo "Logística Comercial”.

Una de nuestras prioridades como docentes es potenciar una formación integral que no sólo capacite al alumnado profesionalmente sino que contribuya al mismo tiempo en dotarle de unas competencias personales de saber trabajar en equipo. Por tanto, nuestros discentes han de adquirir la habilidad de adaptarse y trabajar eficazmente en distintas y variadas situaciones con personas o grupos diversos en la Empresa. Somos conscientes que en la actualidad se demanda en las empresas el uso de técnicas que permitan dominar habilidades sociales como la comunicación, la negociación, la toma de decisiones o la solución de conflictos (Johnson, 2011).

Con la puesta en práctica de la unidad didáctica gestión de Stocks pretendemos: conocer y diferenciar los distintos tipos de stocks que existen; Identificar los elementos que forman el coste total del producto; Saber calcular el coste unitario de adquisición y de fabricación; Planificar cuándo se debe realizar un pedido de un determinado artículo; $y, \quad$ organizar el reaprovisionamiento del stock utilizando el sistema de reposición más aconsejable. Se trata de que el alumnado tenga la oportunidad de analizar los procedimientos de gestión de existencias aplicables en la organización de un almacén.

Y para conseguir estos objetivos, fundamentamos nuestra práctica docente en favorecer el desarrollo personal de manera compartida y colaborativa; estimular la cooperación y apoyo mutuo entre el alumnado; provocar experiencias y vivencias conjuntas que hagan aflorar sentimientos positivos; estimular la interacción comunicativa entre los alumnos/as trabajando en pequeño grupo, por parejas, gran grupo; aprender de los demás y con los demás; y, plantear una metodología activa, participativa y dialógica.

\section{Desarrollo de la experiencia}

La complejidad de la práctica educativa hace necesario que el profesorado asuma el papel de 
investigador; que esté atento a las contingencias del contexto; que se cuestione las situaciones problemáticas de las prácticas; que dé respuesta a las necesidades del alumnado y trate de buscar nuevos enfoques. La enseñanza es un proceso donde tienen lugar simultáneamente múltiples elementos en interacción, lo que hace difícil su indagación y conocimiento (Gimeno, 2010).

Tomamos como referente la idea de investigar la educación como experiencia educativa, es decir, entrar en la experiencia para ver qué pasa, qué nos provoca y qué podemos aprender (Contreras y Pérez de Lara, 2010).

Asumimos un planteamiento de investigación cualitativa en la que los modelos interpretativos cobran importancia (Van Manen, 2003). Estos modelos se basan en la idea de la posibilidad de comprender mejor a las personas a partir de la realidad experiencial. Para ello, el investigador/docente ha de ser reflexivo, intuitivo, sensible al lenguaje y abierto a la experiencia. Es importante acercarse a la experiencia de forma tan imparcial como sea posible. Dado que la experiencia es más inmediata, compleja y ambigua que las descripciones que se hagan de ella, el investigador/docente ha de creer en el poder del lenguaje para hacer inteligible y comprensible aquello que va más allá del lenguaje.

La narración del desarrollo de la experiencia mostrará tres fases /momentos: en la Fase I, el alumnado se centra en la investigación sobre el tema Gestión de Stocks y elaboración de mapas conceptuales. En la fase II realizamos un taller de Diseño-Construcción-Resolución de Problemas sobre Gestión de Stocks, para concluir en la fase III, con la resolución de problemas.

Esta experiencia docente trata de facilitar el aprendizaje significativo de los contenidos.

Investigación sobre el tema gestión de stocks y elabo-ración de mapas conceptuales (Fase I)

El profesor inicia el tema con la formulación de preguntas del tipo de:: ¿Qué es la gestión de stocks?; ¿Cuántos tipos de stocks existen?;¿Cómo los clasificarías?; ¿Qué es el nivel de stock óptimo?; ¿Qué significa una ruptura de stock?; ¿Cómo se comportan las existencias? ; ¿Qué factores influyen en la gestión de stocks? ¡Cuándo deberemos de emitir una orden de pedido?; ¿Qué cantidad debemos solicitar en cada pedido? y ¿Cuántas unidades de cada artículo debemos mantener en stock?

Los alumnos/as de forma individual investigan en el aula de informática sobre los contenidos de la unidad didáctica "Gestión de stocks" y elaboran un mapa conceptual. Se trata de involucrar al alumnado para que busque, observe, seleccione, analice, sintetice y reflexione sobre el tema. En todo este proceso es importante facilitar un ambiente de aprendizaje que ayude al alumnado a construir su propio conocimiento.

A continuación, se desarrollan las siguientes actividades: 1. Búsqueda de información; 2. Elaboración del mapa conceptual; 3. Exposición-Presentación en el aula del mapa conceptual y 4. Construcción grupal de un nuevo mapa conceptual.
A modo de ejemplo presentamos en la Figura 1 el mapa conceptual elaborado por el grupo.

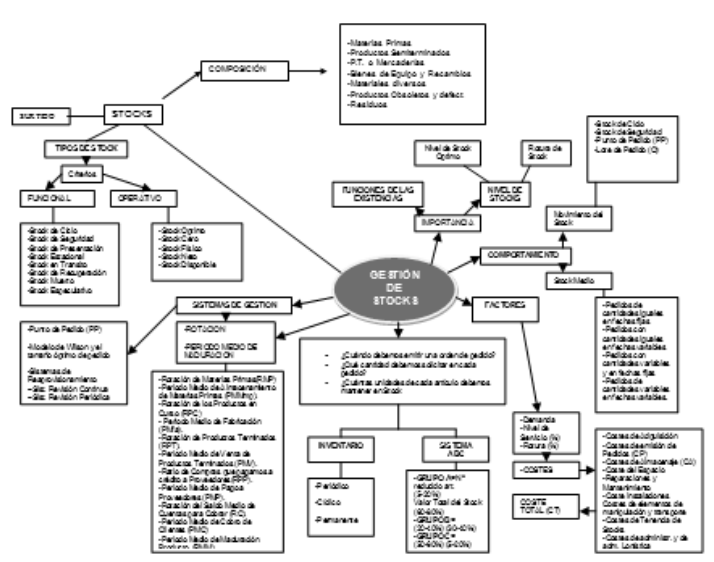

Figura 1. Construcción grupal del mapa conceptual.

Taller aprender juntos: Diseño-construcciónresolución de problemas sobre gestión de stocks (Fase II)

Búsqueda de fórmulas de gestión de stocks y elaboración ficha-registro individual de fórmulas.

En el aula de informática cada alumno/a ha de buscar formulas sobre la gestión de stocks y registrarlas en una ficha-documento (concepto/Fórmula/ Descripción). Posteriormente, expresa para qué sirve la fórmula, la fórmula general y la especificación de cada uno de los elementos que la componen.

Exposición individual al grupo y elaboración ficharegistro-grupal.

Cada alumno/a expone y presenta su trabajo al gran grupo utilizando los recursos tecnológicos más apropiados: presentación power, prezi, etc. A partir de las presentaciones individuales, se inicia el trabajo de gran grupo y pequeño grupo. Cada pequeño grupo realiza una comparativa con sus propias fórmulas con el objetivo que todos los alumnos/as tuvieran la mayoría de fórmulas en su ficha-documento.

Cambiamos la dinámica y la organización social del alumnado para iniciar así el trabajo en gran grupo. Se genera debate y dialogo en el aula, surgen preguntas, etc. El alumnado tiene la oportunidad de valorar, discutir y argumentar sobre las diferentes formas que una fórmula puede presentar, asumiendo que todas son igualmente válidas.

Búsqueda y resolución de problemas de ges-tión de stocks. Elaboración documento-ficha con 10 problemas y sus resoluciones.

En este momento recurrimos a la organización del alumnado en parejas. El trabajo conjunto en pares y la ayuda entre iguales es importante para la construcción conjunta del conocimiento (Mercer, 1997).

El profesor que conoce bien la complejidad del aula y los niveles de conocimiento del alumnado en el tema que nos ocupa, organiza estas parejas tratando de posibilitar y favorecer el intercambio de saberes ya que las capacidades, habilidades y destrezas son diferentes. Así, el alumnado tiene la oportunidad de tomar conciencia de que el trabajo grupal requiere cooperación 
y colaboración. Por tanto, ha de asumir que de la responsabilidad individual dependerá el éxito del equipo de trabajo.

Hemos de explicitar que es importante que antes de empezar la actividad en sí, el profesor especifique y detalle en que consiste la tarea y qué objetivos pretende. Se trata de una cooperación entre pares. En palabras de Cabrera (2008: 610) "las interacciones entre alumnos, debidamente organizados por el docente, pueden convertirse en oportunidades de aprendizaje".

La actividad consiste en que cada pareja tiene que buscar diez problemas-modelo en el aula de informática e intentar resolverlos con la ayuda del documento-ficha en el que están registradas todas las fórmulas referentes a gestión de stocks.

En este momento tienen también que elaborar un Documento en el que registrarán diez problemas con sus correspondientes resoluciones (fase de entrenamiento).

Creación - diseño y resolución de problemas de gestión de stocks. Elaboración de documento-ficha.

Se trata de involucrar al alumnado para que tenga iniciativa y motivarlo hacia la consecución de un logro, como es la resolución de su propio problema. Por parejas, cada alumno/a resolverá los problemas diseñados por su propio compañero y posteriormente, analizarán y compararan los resultados. Se elabora un informe común con los veinte problemas resueltos y se diseña un documento paralelo con los veinte problemas sin resolver.

Intercambio de pares -resolución de problemas con apoyo entre iguales.

El objetivo es que cada alumno/a resuelva los problemas diseñados por su par. Ante las dudas planteadas en la resolución de cada uno de los problemas su propio compañero será el que le ayudará en la resolución del mismo.

Resolución individual. Problemas gestión de stocks (Fase III)

En esta fase los grupos de trabajo están ya consolidados y han vivenciado lo importante que es trabajar juntos para avanzar en el proceso de aprendizaje.

Intercambio de problemas a nivel de gran grupo y resolución individual de los mismos.

A través de sus correos electrónicos cada grupo envía su Informe de problemas resueltos al resto de compañeros/as, así todo el alumnado del grupo- clase tiene un dossier de problemas resueltos a su disposición para practicar a nivel individual en la resolución de problemas de gestión de stocks.

\section{Metodología}

Asumimos que "Investigar es siempre cuestionar el modo en que experimentamos el mundo, querer conocer el mundo en el que vivimos en nuestra calidad de seres humanos" (Van Manen, 2003:23).

En nuestro caso, la investigación cualitativa nos ofrece multitud de enfoques diferentes relacionados siempre con la explicación de fenómenos sociales que son vistos desde el interior de los mismos. Se trata de acercar una realidad sobre grupos, individuos a través de sus experiencias, historias de vida, etc. Y lo hacemos, analizando sus interacciones, comunicaciones, progresos, documentos y experiencias (Angrosino, 2012). Pretendemos saber de manera más profunda, cómo construyen el mundo desde el ámbito que vamos a observar: el aula de Formación Profesional (FP).

Desde el contexto de FP necesitamos herramientas y recursos didácticos para poder dar respuesta a la diversidad de estudiantes que conviven en un mismo grupo. El aprendizaje colaborativo se nos presenta como una alternativa eficaz para ello. Es importante explicitar que, en nuestro caso, nos situamos ante un grupo de alumnos bastante heterogéneo, de características complejas donde existe mucha diversidad de ritmos de aprendizaje y con carencias de hábitos de estudio importantes. En general, presentan poca motivación e interés para el aprendizaje del curriculum académico del ciclo formativo.

Ante esta realidad, los docentes nos planteamos reflexionar sobre nuestra práctica docente y centrar la enseñanza en el alumnado tratando de despertar curiosidad en el discente con la intención de implementar un aprendizaje significativo y relevante desde la construcción del conocimiento en el aula y la transición al mundo del trabajo.

Asumimos que la investigación ha de posibilitar la transformación de la acción educativa.

La función del docente es de guía, ayuda y dinamizador del proceso. El profesor, en esta clase, abre caminos, despierta intereses, observa, camina con ellos, aprende de ellos,..etc. Estamos de acuerdo con Bruner que si la labor del profesor consistiera en explicar las lecciones, estaría matando la capacidad de investigar del alumnado (Bruner, 1997).

Asumiendo esta perspectiva, el profesor no explica, sino que complica poco a poco, asombra a los alumnos, cuando ellos mismos no son capaces de dudar de la absoluta realidad de sus conocimientos. Es preciso admitir la crítica como una manera de aprender y generar contextos que lo posibiliten.

\section{Resultados y conclusiones}

En cuanto a resultados y conclusiones, constatamos que esta forma de trabajar ha mejorado las relaciones personales dentro y fuera del aula. Se han creado canales de comunicación que han propiciado un clima de aprendizaje positivo. El alumnado ha adquirido habilidades y conocimientos para gestionar compras y aprovisionamientos. Asimismo, han aprendido estrategias varias para resolver problemas y saben cuándo se debe emitir una orden de pedido y las unidades de cada artículo que debemos mantener en stock.

Inicialmente los alumnos/as sentían desconfianza al realizar las tareas propuestas, aunque el profesor les había explicado el proceso a seguir. Constatamos que no están acostumbrados a trabajar colaborativamente sino a formar grupos de trabajo que se distribuyen de una forma individual la tarea a realizar. Aparentemente no se avanza y continuamente demandan ayuda porque no tienen claro lo que se va a hacer. Poco a poco van valorando y toman conciencia de lo que hacen y dan sentido al hacer. Ellos mismos se motivan por los logros alcan- 
zados y sienten que han aprendido a resolver problemas y sobretodo, a entenderlos.

Constatamos en el desarrollo de esta experiencia que a medida que ha transcurrido el curso escolar los alumnos estaban más integrados en la dinámica de clase y con una actitud más participativa. Además desde los debates y reflexiones planteados hemos llegado a construir conocimiento común que les ha ayudado a tomar conciencia de su propia potencialidad para actuar como sujetos y de la necesidad de fomentar el aprendizaje de la participación y la convivencia entre las personas.

A nivel teórico el diseño del mapa conceptual de la unidad didáctica les ha permitido identificar y relacionar conceptos favoreciendo el aprendizaje significativo de los contenidos referidos a la gestión de stocks, tales como: Tipos de stocks; Funciones de las existencias; Nivel de Stocks y su comportamiento; Factores y Sistemas de Gestión de stocks, etc.

El rol del profesor/a en este tipo de actividades es diferente, habiendo de planificar de forma clara y concisa cada una de las tareas, indicando al alumnado en qué va a consistir su trabajo así como los criterios de evaluación. No se trata de "aprender compitiendo" sino "aprender compartiendo". De esta forma se adapta un currículum común a las características individuales de cada uno de los alumnos/as y la cooperación entre iguales contribuye a generar un clima/ambiente de aula más participativo. El profesor/a guía, motiva, orienta, concilia, media, coordina, etc. Cuando el grupo de pares se encuentra con dificultades, el profesor hace sugerencias de cómo se podría llevar a cabo la tarea, impulsando así la iniciativa de sus alumnos/as, motivándoles hacia la consecución de logros y fomentando la colaboración.

El docente reflexiona analizando su práctica y promoviendo aprendizajes significativos ajustados a la diversidad de necesidades o intereses en que se involucran los alumnos, evitando apoderarse de la palabra y convertirse en un transmisor de información para no caer en la enseñanza verbalista o unidireccional (Díaz-Barriga, 2005).

La reflexión del docente despierta innovaciones pedagógicas en el aula y como decía Freire (2008) una de las tareas más importantes de la práctica educativa crítica es propiciar las condiciones en que los educandos en sus relaciones unos con los otros y todos con el profesor intentan la experiencia profunda de asumirse. Asumirse como ser social e histórico, como ser pensante, comunicante, transformador, creador y realizador de sueños.

\section{Referencias}

Angrosino, M. (2012). Etnografia y observación participante en Investigación Cualitativa. Madrid: Morata.

Anijovich, R., Mora, S. (2010). Estrategias de enseñanza: otra mirada al quehacer en el aula. Buenos Aires: Aique.

Bruner, J. (1997). La educación, puerta de la cultura. Madrid: Visor.

Cabrera, E. P. (2008). La colaboración en el aula, más que uno más uno. Revista Latinoamericana de Psicología, 41 (3). Colombia: Fundación Universitaria Konrad Lorenz.
Contreras, J. y Pérez de Lara, N. (comps). (2010). La experiencia y la investigación educativa. Madrid: Morata.

Díaz Barriga, F. et all.(2005). Estrategias docentes para un aprendizaje significativo. México: McGraw-Hill.

Edwards, D y Mercer, N. (1987). El conocimiento compartido. Barcelona: Paidós.

Freire, P. (2008). Pedagogía de la autonomía. Saberes necesarios para la práctica educativa. Buenos Aires: Siglo XXI.

Gimeno Sacristán, J. [Comp.] (2010). Saberes e incertidumbres sobre el curriculum. Madrid: Morata.

Johnson, S. (2011). Las buenas ideas. Una historia natural de la innovación. Madrid: Ediciones Turner.

Ley Orgánica 2/2006, de 3 de mayo, de Educación (LOE). Boletín Oficial del Estado, 4 de mayo de 2006, núm. 106, p. 17158-17207.

Ley Orgánica 8/2013, de 9 de diciembre, para la Mejora de la Calidad Educativa (LOMCE). Boletín Oficial del Estado, 10 de diciembre de 2013, núm. 295, p. 97858-97921.

Mercer, N. (1997). La construcción guiada del conocimiento. Barcelona: Paidós.

Morin, E. (2011). Los siete saberes necesarios para la educación del futuro. Barcelona: Paidós.

Pérez Gómez, A. I. (2012). Educarse en la era digital. Madrid: Morata.

Real Decreto $1651 / 94$, de 22 de julio por el que se establece el título de Técnico Superior en Gestión Comercial y Marketing. Boletín Oficial del Estado (BOE), 28/9/94.

Van Manen, M. (2003). Investigación educativa y experiencia vivida. Barcelona: Idea books.

Wells, G. (2001). Indagación dialógica. Hacia una teoría y una práctica socioculturales de la educación. Barcelona: Paidós

Woods, P. (2011). La escuela por dentro. Madrid: Ministerio de Educación y Ciencia. 